Voix et Images

volxetimages

\title{
À propos de « Bêtes et mari » de Jacques Ferron
}

\section{Guy Monette}

Volume 16, numéro 1 (46), automne 1990

Les correspondants littéraires d'Alfred DesRochers

URI : https://id.erudit.org/iderudit/200878ar

DOI : https://doi.org/10.7202/200878ar

Aller au sommaire du numéro

Éditeur(s)

Université du Québec à Montréal

ISSN

0318-9201 (imprimé)

1705-933X (numérique)

Découvrir la revue

Citer cet article

Monette, G. (1990). À propos de « Bêtes et mari » de Jacques Ferron. Voix et Images, 16(1), 122-127. https://doi.org/10.7202/200878ar d'utilisation que vous pouvez consulter en ligne.

https://apropos.erudit.org/fr/usagers/politique-dutilisation/ 


\section{À propos de «Bêtes et mari * de Jacques Ferron}

\section{par Guy Monette, Collège militaire royal, Kingston}

"Bêtes et mari " 1 : une simple fantaisie dans laquelle Jacques Ferron se livre à la joie des mots et des images. Que demander de plus à un conte? Ainsi, Jean-Pierre Boucher ne traite pas de ce conte dans une étude, par ailleurs fort intéressante, et il le range sous une rubrique qui regrouperait des textes où Ferron invite simplement le lecteur à s'évader ${ }^{2}$. Certes, une sensualité mutine émaille allégrement le texte; mais est-ce là motif à faire intervenir les ridicules nippes freudiennes ${ }^{3}$ ? Même Bruno Bettelheim affirme, dans The Uses of Enchantment, que le conte de fées suscite surtout un plaisir esthétique:

Le plaisir que nous ressentons, l'émerveillement qui nous emporte lorsque nous nous abandonnons aux contes de fées, découle non pas tant de la signification psychologique d'un conte (bien qu'elle n'y soit pas étrangère) que des qualités littéraires de ce conte en tant qu'œuvre d'art. ${ }^{4}$

La lecture de "Bêtes et mari " présentée ici gomme, volontairement mais à contre-cœur, la valeur esthétique de ce conte et, la qualité du style ferronien. En ce sens, elle est, selon la terminologie de Tzvetan Todorov, une projection interprétative. C'est également ce genre de projection interprétative que pratique Bettelheim dans The

1 Jacques Ferron, Contes, Montréal, HMH, 1968, p. 104-105.

2 Jean-Pierre Boucher, les “Contes» de Jacques Ferron, Montréal, l'Aurore, 1974, p. 101.

3 Jean Marcel, Jacques Ferron malgré lui, Montréal, Parti pris, 1978, p. 56 (Frères chasseurs).

4 The delight we experience when we allow ourselves to respond to a fairy tale, the enchantment we feel, comes not from the psychological meaning of a tale (although this contributes to it) but from its literary qualities - the tale itself as a work of art. (Bruno Bettelheim, The Uses of Enchantment. The Meaning and Importance of Fairy Tales, New York, Alfred A. Knopf, 1976, p. 12. Ma traduction.) Ce livre a été traduit en français sous le titre quelque peu trompeur de Psychanalyse des contes de fées (traduction de Théo Carlier, Paris, Robert Laffont, 1976). La Psychanalyse et les contes de fées eút été une traduction plus juste. De l'utilité des contes de fées ou Enchanter pour libérer aurait aussi mieux correspondu au contenu du livre. Quoi qu'il en soit, on pourra consulter cetté traduction. 
Uses of Enchantment. Ce dernier démontre en effet que les contes de fées peuvent aider les enfants qui les entendent à résoudre les conflits auxquels ils font face au cours de leur évolution psychique, en leur fournissant des modèles qui soient à leur portée, qui leur permettent d'exprimer et de vivre par procuration leurs fantasmes, tout en leur indiquant que ces conflits ne sont pas anormaux et sont susceptibles de trouver une conclusion heureuse. Comme le travail de Bettélheim avait un but éducatif et voulait montrer l'utilité des contes de fées traditionnels, il est axé beaucoup plus vers la signification que peut avoir le conte pour son lecteur ${ }^{5}$ (dans ce cas, l'enfant) que sur sa fonction par rapport au créateur (dans ce cas, la tradition ${ }^{6}$ ).

De toute évidence, les contes de Ferron ne sauraient être assimilés à des contes de fées, quoique "Bêtes et mari", par rapport à d'autres contes ferroniens, s'apparente davantage à ce genre. Il va de soi également qu'un lecteur peut, consciemment ou inconsciemment, utiliser certains aspects d'un conte d'une manière particulière et leur accorder une signification étrangère aux intentions du créateur de ce conte (dans la mesure où les dites intentions sont identifiables). Bettelheim donne d'ailleurs plusieurs exemples de ce que les enfants élaborent à partir des contes de fées. Toutefois, les théories de Bettelheim pourraient jeter un éclairage nouveau sur "Bêtes et mari ". Sans doute le genre d'analyse pratiqué ici se situe-t-il, dangereusement, à la frontière de la psychanalyse et de la critique littéraire. En outre, les manifestations qui y seront illustrées relèvent à la fois de l'inconscient individuel de l'auteur (et du critique) et de l'inconscient collectif (au sens donné à cette expression dans la note 6). Une telle analyse néglige aussi le problème des liens qu'entretiennent la psyché d'un auteur et la tradition culturelle dans laquelle s'inscrit cet auteur. On jugera de cette expérience, toute empirique et consciente des risques qu'elle implique, à la valeur dè ses résultats.

Il ne serait pas approprié de reprendre les conclusions ou le détail du travail de Bettelheim et l'on se rapportera, pour plus d'informations, à The Uses of Enchantment, particulièrement au dernier chapitre (p. 277-310) qui traite de contes du cycle du conjoint animalisé. Guidé par sa théorie qui veut que les contes de fées peuvent exprimer les hantises inconscientes de l'enfant tout en leur offrant une solution, Bettelheim voit dans les contes de ce cycle une illustration du fait que l'enfant, pour conquérir la maturité psychique, doit à la fois abandonner sa conception trop égoïste du plaisir et exorciser les peurs que lui inspire la sexualité; la transformation d'un animal quelque peu

5 De là le caractère trompeur du titre de la traduction française auquel il est fait allusion à la note précédente.

6 Cette tradition apparait chez Bettelheim comme la somme de l'expérience psychique des divers individus qui y contribuèrent et correspond ainsi à une espece d'inconscient collectif induit des inconscients individuels à travers l'histoire. 
répugnant en un partenaire sexuel humain désirable serait l'aboutissement symbolique de cette conquête de la sexualité.

Abordé dans cette optique, le texte de "Bêtes et mari" devient particulièrement signifiant. Déjà par son titre, ce conte évoque le cycle du conjoint animalisé; ce que confirme la lecture du texte. De plus, le pluriel de "Bêtes" joint au singulier de "mari» laisse soupçonner une possibilité d'évolution des bêtes puisqu'il faut établir un rapport entre plusieurs bêtes et un seul mari.

Le conte s'ouvre sur une situation dans laquelle le narrateur masculin est aux prises avec une réalité qu'il contrôle peu et où il a pour guide une initiatrice savante (maîtresse d'école). L'école privée dont il est question désigne métaphoriquement un bordel; mais elle peut aussi être une représentation du but de la pulsion sexuelle. Ce but éloigné dans l'espace (au bout du rang) témoignerait de l'effort qu'exige la pulsion. Le narrateur entre alors en contact avec des faits certes fascinants, mais qui représentent quelques périls dont le moindre est que la réalité en question n'est jamais clairement identifiée (lune de Paris, dessous de la vie, mauvaise réputation, mélancolique, triste, pensif, gêné). On aura évidemment reconnu la prostituée sous l'apparence de la maîtresse au sobriquet si juste d'allumette. Mais on peut, sous son aspect de veuve plus âgée, de mauvaise réputation, dotée d'un pouvoir ou d'un savoir supérieurs, voir en elle un avatar de la sorcière du conte traditionnel qui souvent tente et trompe l'enfant mais qui, en outre, métamorphose initialement un humain en animal, faisant ainsi de lui un objet sexuel répugnant.

S'il n'y a pas d'animalisation directe du narrateur, un animal apparaît tout de même (l'éléphant). Ce qu'il représente, surtout l'aspect sexuel de la personnalité mais ausi, évidemment, l'organe sexuel (la trompe), n'est pas intégré par le narrateur puisque l'animal l'accompagne et lui causera des ennuis à la fois sociaux (réaction des gens de Fontarabia) et personnels (l'honorée-gonorrhée). L'incongruité de la présence de l'éléphant et son poids indiqueraient de plus à quel point le narrateur est débordé par la pulsion. En outre, si le symbolisme phallique est ici patent (la trompe de l'éléphant, la corne de la licorne, le rat ${ }^{7}$ ), les dimensions décroissantes de l'animal symbolique indiquent à la fois que la sexualité prend des proportions moins envahissantes (probablement est-elle plus contrôlée), que le fantasme de la taille du pénis (obsession masculine qui camoufle une peur d'être sexuellement inadéquat) se résorbe, mais aussi que le rôle sexuel du mâle est dévalorisé graduellement jusqu'à ce que se produise l'intégration de la sexualité à la personnalité.

La poussée sexuelle initiale aboutit à une punition: l'éléphant tombe malade, de la trompe, bien entendu. L'éléphant a fait un geste

7 On traitera du (et des) chien(s) plus avant. 
dont il ne pouvait contrôler toutes les conséquences et le narrateur est exposé à une réalité qui risque de l'écraser. Le recul devant la pulsion s'impose donc et le moi-éléphant est puni par où il a péché. D'accompagnateur qu'il était, l'éléphant devient alors presque fusionné au narrateur puisque l'animal est soigné par un médecin, et non par un vétérinaire, et que c'est le narrateur qui se dit guéri.

La leçon porte et l'éléphant disparaît pour laisser place à un autre animal symbolique déjà plus intégré et contrôlé par le narrateur puisque ce dernier chevauche la licorne alors que l'éléphant l'accompagnait et était libre de ses mouvements. Cette licorne, en plus d'être clairement une représentation phallique (la bête et sa corne unique sont à l'enfourchure puisque le narrateur chevauche l'animal), s'associe alors à l'abstinence sexuelle. Il y aurait probablement, dans ce cas, un lien à établir avec l'onanisme par opposition à une relation sexuelle. En effet, la licorne, traditionnel symbole de pureté, ne se laisse approcher que par un être humain vierge et vient poser sa tête dans le giron de ce dernier pour se laisser caresser.

Devant la poussée de la sexualité, qui s'avérait trop menaçante, le narrateur a clivé la sexualité en deux composantes: une sexualité contrôlée (la licorne) qui se ramène à l'abstinence et qui a été intégrée par le narrateur et une autre sexualité débridée mais non intégrée par le narrateur qui s'incarne dans les trois (ce nombre indique sa puissance) chiens presque enragés et rappellent la menace de l'expérience précédente. La puissance destructrice maléfique des chiens est des plus claires. D'ailleurs, l'admission ou l'exclusion de l'enceinte sacrée du presbytère confirme, et pour la licorne et pour les chiens, le sens du clivage de la sexualité du narrateur.

Pour que le clivage de la personnalité disparaisse, il faut que l'énergie sexuelle soit canalisée autrement, que ses deux composantes soient conciliées et intégrées; sinon, le narrateur serait condamné à chevaucher la licorne ou à être détruit par les chiens. La solution proposée par le curé serait idéale si la relation maritale maintenait l'intégrité du narrateur dans un rapport d'égal à égale avec la partenaire sexuelle comme c'est le cas au début de leur mariage. En effet, la comparaison contrebalançant la métaphore, l'animalisation partielle de l'épouse annule celle de l'époux: Ma femme grasseye comme une souris de Charlesbourg. Elle m'appelle son gros rat blanc. Le mot souris désigne l'épouse en son aspect sexuel et apparente cette dernière à la maîtresse du début du conte tout en la rendant moins puissante. De même, l'adjectif gros, tout en soulignant l'obsession phallique, fait contrepoids au pouvoir maléfique des grands chiens, symbole de la sexualité débridée.

La sexualité n'était cependant entièrement intégrée qu'en apparence puisque le narrateur se transforme vraiment par la suite en animal, ce qui le dévalorise dans son rôle sexuel et le rend 
superflu. Le conte suscite alors un modèle humain plus approprié au contexte: un amant surgit littéralement du passé sexuel de l'épouse. Il peut combler l'épouse (Ils sont contents de se revoir) dont l'intérêt est marqué par une superbe et coquine métaphore (Ma femme l'époussette). Le mari-rat dévalorisé risque d'être totalement exclu de la relation avec l'objet sexuel dans laquelle il s'était cantonné à un rôle diminué qui ne satisfaisait probablement que lui seul: quoi de plus reposant après l'éléphant et la licorne! Je suis content, je suis heureux. Il s'agit d'une nette régression par rapport aux événements antérieurs et le narrateur devient un objet passif dont tout le bienêtre dépend du bon vouloir de l'épouse: Ma femme fait de moi ce qu'elle veut. S'agirait-il là d'une relation infantile avec un possible substitut maternel?

Amant et rat représenteraient dans ce cas deux facettes de la sexualité masculine: l'une "normale» et typique de l'adulte, l'autre dévalorisée et infantile. Il y a lutte entre ces deux facettes pour assurer la possession de l'objet sexuel, comme l'indique le côtoiement de l'amant et du rat dans le lit de l'épouse. Le narrateur dévalorisé et régressif risque alors l'exclusion comme en témoigne la transformation de l'amant en chat qui menace de dévorer le rat-mari. L'on pourrait également évoquer à propos de cet amant une figure paternelle contre laquelle le fils lutterait; la disparition possible du rat phallique serait en ce sens une menace de castration.

Le narrateur comprend alors que la "normalité" est la seule solution valable (c'est d'ailleurs celle qu'a choisie l'épouse en faisant appel à l'amant) et il réagit en se transformant en chien et en chassant le chat-amant pour s'assurer de la conquête de l'objet sexuel. La transformation du rat en chien inverse le processus de dévalorisation (dimension croissante de l'animal symbolique) et marque un retour vers la pulsion sexuelle initiale puisque ce chien aboyant est de même espèce que les trois chiens noirs d'une séquence précédente. Toutefois la pulsion, plutôt que de menacer le narrateur, vient à ce moment à son aide: il l'a canalisée à son profit. La sexualité débridée a, elle aussi, été intégrée par le narrateur car c'est lui qui devient chien. De plus, le geste du chien-mari qui pose son museau sur les genoux de l'épouse transcende l'image mythique de la licorne posant sa tête sur les genoux de la vierge. (Puisque ces deux animaux sont des symboles phalliques, l'action décrite ne peut qu'évoquer une relation sexuelle.) Cette vierge est ici l'épouse qualifiée d'infidèle, c'est-àdire que, sexualisée, elle assume la mauvaise réputation de la veuve du début du conte. L'épouse intègre ainsi deux aspects de la sexualité féminine comme le chien-mari concilie les aspects débridés (les trois chiens noirs) ou trop contrôlés (la licorne) de la sexualité masculine.

Le chien-mari peut repousser son rival puisqu'il a incorporé ce que représentait le chat-amant; le clivage entre la sexualité "normale" 
et la sexualité dévalorisée n'a plus sa raison d'être. Le mari-chien est donc plus fort que l'amant devenu chat. Le narrateur aurait, selon une autre perspective, réussi à supplanter le père dans un nouveau triangle œdipien et éliminerait ainsi et le père et le triangle. À ce propos, il faut noter que les figures d'autorité (médecin et curé), si elles aident le narrateur (guérison et conseils), ne lui fournissent cependant qu'une solution incomplète (la licorne indique la guérison, mais n'apporte pas la satisfaction; le mariage ne règle pas le problème et en suscite de nouveaux). Le père, figure d'autorité, n'aurait pas intérêt à ce que le fils atteigne une autonomie qui risquerait de diminuer le prestige du père en faisant du fils un égal. Selon Bruno Bettelheim, les contes de fées, par exemple "Blanche 'Neige ", aident les enfants à accepter le fait que le parent du même sexe puisse être envieux du développement de l'enfant et cherche à le contrer ${ }^{8}$.

La dernière étape de l'évolution du mari et de sa conquête de la sexualité "normale" est alors facile à franchir et il devient homme "normal" à l'incitation d'une partenaire "normale": Grand fou, ditelle, veux-tu bien être un homme? Le grand de cette expression contrebalance la relation infantile antérieure et l'épouse reconnait, par lui, la valeur sexuelle du narrateur et l'inscrit dans une relation humaine "normale". Le narrateur peut ainsi établir une relation d'égal à égale, posséder l'objet en le satisfaisant et intégrer tous les aspects de sa sexualité.

Les contes de fées du cycle du conjoint animalisé présentent d'ordinaire le point de vue féminin de la conquête et de l'intégration de la sexualité. C'est ce point de vue féminin qu'on retrouve dans la chanson folklorique Mon père m'a donné un mari; il y est question d'un mari petit comme une souris, qui se perd dans le lit de son épouse et que menace un chat. Ferron s'en est probablement inspiré pour son conte. "Bêtes et mari" offre le point de vue masculin du même processus puisque le narrateur y est un homme. La situation initiale d'apprentissage du conte (J'allais à l'école) trouve sa conclusion heureuse à la fin du récit (Ce fut ainsi [...] que je devins un bon mari). Ainsi, comme dans les contes de fées, hautement moraux en dépit de leur apparente amoralité, tout est bien qui finit bien.

8 "The jealous Queen in Snow White and the myth of CEdipius", The Uses of Enchantment, op. cit., en particulier p. 194-199. 